Editorial

\title{
Gender effect on migraine: nature or culture?
}

\author{
Rocco Salvatore Calabrò ${ }^{1, *}$ \\ ${ }^{1}$ Neurorehabilitation Unit, IRCCS Centro Neurolesi “Bonino-Pulejo", 98124 Messina, Italy \\ *Correspondence: salbro77@tiscali.it (Rocco Salvatore Calabrò)
}

\section{DOI:10.31083/j.jin2101018}

This is an open access article under the CC BY 4.0 license (https://creativecommons.org/licenses/by/4.0/).

Submitted: 7 January 2022 Revised: 11 January 2022 Accepted: 14 January 2022 Published: 28 January 2022

Migraine is a very common disorder, affecting about $12 \%$ of adult populations in Western countries. Its incidence is threefold higher in women than in men, with a greater occurrence among women during reproductive years. This disparity is believed to be related to the different sexual hormones produced in male and females, where estrogens and progesterone are understood to play a pivotal role in causing the disease [1]. Headache can be triggered any time there is a fluctuation in estrogen levels: a decrease of plasma estrogen can trigger migraine attacks while an increase seems to be protective. There is a worsening of headache around the time of menses, while in pregnancy hormonal headaches tend to be less frequent, increasing again after delivery. Fluctuations are also present in perimenopause and menopause [1, 2]. In addition, oral contraceptives and hormone replacement therapy may cause migraine attacks.

The pathophysiological mechanism of migraine is complex and involves both the peripheral trigeminovascular system and the interaction between sexual hormones and many other peptides, including oxytocin and calcitonin generelated peptide (CGRP) [1]. The latter seems to have a role in the development and maintenance of migraine attacks, considering the favorable effects of the CGRPergic monoclonal antibodies in the prophylaxis of chronic migraine [3].

Nonetheless, the abnormal difference in prevalence, frequency and intensity of migraine between sexes cannot be explained by hormonal causes alone. Other relevant biological and behavioral differences must be taken into account.

A recent study has demonstrated that women suffering from migraine had significantly more relational and social problems, including less feelings of being understood by family, friends, and partners, as well as higher anxiety, pain and depression symptoms than men [4]. As such, social stress may exacerbate migraine attack incidence and intensity.

The genetic load of various gene polymorphisms found to be more prevalent in migraines can cumulatively impact risk and determine a critical attack threshold, which may be modulated by external (psychosocial stress, mood, anxiety and preventive therapies) and internal factors (hormonal status, mood, and anxiety) [5]. Triggers, such as alcohol, specific foods, or stress, are more likely to induce an attack when the migraine threshold is lowered, e.g., by estrogen level varia- tions, and the trigeminovascular system is activated [5]

In recent years, increasing attention has been paid to the management of migraine in patients belonging to sexual and gender minority populations, including but not limited to individuals who identify as lesbian, gay, bisexual, asexual, transgender, queer, and/or intersex.

Numerous policy recommendations have been made to prevent discriminatory practices, including discrimination in the workplace, harassment in educational settings, and forced treatment or conversion therapy of sexual and gender minorities. Nevertheless, stress, stigma, discrimination, and socioeconomic disadvantages persist in affecting health outcomes and overall perception of health in these minority groups, particularly in the transgender population $[6,7]$. Disclosing sexual orientation and gender identity in the primary care setting is fundamental because a comfortable dialogue about the patient's sexual identity, orientation and relationships can help to personalize healthcare assistance, with a better quality of care [8]. Indeed, those within these minority groups often present with psychiatric comorbidities, such as anxiety, depression, and other stress-related mental disorders [9] that can affect migraine frequency and severity. This could be why preliminary data evidence higher rates of migraine in transgender as compared to cisgender individuals [7]. As hormones may affect both mood and migraine incidence, it is possible that gender-affirming hormone therapy may play a role in precipitating migraine through both a direct and indirect mechanism $[6,7]$. In summary, it may be that gender may affect migraine through a complex interaction of biological and behavioral factors.

Nature and culture are popularly seen as opposite concepts: what is the result of nature cannot be the result of human intervention and behavior, and vice versa. Theories concerning the evolutionary development of human beings suggest that culture is part of the ecological niche within which our species thrived, thus rendering culture fundamental for the biological development of a species.

Along these lines, migraine is due to 'nature', given that its biological components, including sex, age, and sexual hormones, play a pivotal role in determining the disease. However, migraine is also related to 'culture', as women are more easily subjected to social stress and relational problems [4], 
and sexual minorities suffer from stigma and various discrimination [9].

The pathophysiology of migraine is a complex and still incompletely understood issue. Further investigation of the gender effect is needed to shed more light on this common yet disabling disease.

\section{Ethics approval and consent to participate}

Not applicable.

\section{Acknowledgment}

Not applicable.

\section{Funding}

This research received no external funding.

\section{Conflict of interest}

The author declares no conflict of interest. RSC is serving as Guest Editor of this journal. We declare that he had no involvement in the peer review of this article and has no access to information regarding its peer review. Full responsibility for the editorial process for this article was delegated to RF.

\section{References}

[1] Krause DN, Warfvinge K, Haanes KA, Edvinsson L. Hormonal influences in migraine-interactions of oestrogen, oxytocin and
CGRP. Nature Reviews Neurology. 2021; 17: 621-633.

[2] Al-Hassany L, Haas J, Piccininni M, Kurth T, Maassen Van Den Brink A, Rohmann JL. Giving Researchers a Headache - Sex and Gender Differences in Migraine. Frontiers in Neurology. 2020; 11: 549038.

[3] González-Hernández A, Marichal-Cancino BA, Villalón CM. The impact of CGRPergic monoclonal antibodies on prophylactic antimigraine therapy and potential adverse events. Expert Opinion on Drug Metabolism and Toxicology. 2021; 17: 1223-1235.

[4] Neumeier MS, Pohl H, Sandor PS, Gut H, Merki-Feld GS, Andrée C. Dealing with Headache: Sex Differences in the Burden of Migraine- and Tension-Type Headache. Brain Sciences. 2021; 11: 1323.

[5] Khan J, Asoom LIA, Sunni AA, Rafique N, Latif R, Saif SA, et al. Genetics, pathophysiology, diagnosis, treatment, management, and prevention of migraine. Biomedicine \& Pharmacotherapy. 2021; 139: 111557.

[6] Pace A, Barber M, Ziplow J, Hranilovich JA, Kaiser EA. Gender Minority Stress, Psychiatric Comorbidities, and the Experience of Migraine in Transgender and Gender-Diverse Individuals: a Narrative Review. Current Pain and Headache Reports. 2021; 25: 82.

[7] Hranilovich JA, Kaiser EA, Pace A, Barber M, Ziplow J. Headache in transgender and gender-diverse patients: A narrative review. Headache: The Journal of Head and Face Pain. 2021; 61: 10401050.

[8] Calabrò RS, Quattrini F. Disclosing Sexual Orientation to Healthcare Providers: still a Taboo or Time for a Change? Journal of the National Medical Association. 2018; 110: 596-597.

[9] Plöderl M, Tremblay P. Mental health of sexual minorities. A systematic review. International Review of Psychiatry. 2015; 27: $367-385$. 